6B.003 UNDERREPORTING OF DROWNING IN NIGERIA: A RETROSPECTIVE SURVEY OF NATIONAL NEWSPAPER REPORTS

1,2Anthony Mckeever*, 3,4Shafkat Hossain. 'Liverpool School of Tropical Medicine, Liverpool, UK; ${ }^{2}$ University of Liverpool Medical School, Liverpool, UK; ${ }^{3}$ International Drowning Research Centre, Bangladesh, Mohakhali, Bangladesh; ${ }^{4}$ Centre for Injury Prevention and Research, Bangladesh, Mohakhali, Bangladesh

10.1136/injuryprev-2021-safety. 154

Background According to the WHO, over 300 people drown every week in Nigeria. However, this figure excludes drownings caused by watercraft accidents and flood disasters. Research indicates that the WHO vastly underestimates drowning mortality because of this. Understanding the true extent of the drowning crisis in Nigeria is crucial to engaging stakeholders to implement prevention strategies.

Aim To assess the extent of underreporting of drowning mortality by the WHO in Nigeria.

Methods Vanguard news online database was searched for articles reporting fatal drowning incidents between 20092019. The causes or circumstances of each incident were extracted to create a sample of drowning victims. Causes of drowning were coded according to the ICD-10, with inclusion of all-cause drowning codes. Drownings caused by codes classified by the WHO as drowning deaths were compared to allcause drowning deaths to estimate the extent of underreporting.

Results 346 drowning victims were sampled from 98 articles. $54.9 \%(n=175)$ of drownings were caused by watercraft accidents. Flood disasters, intentional causes and land transport accidents caused 5.3\% $(n=17), 1.5 \%(n=5)$ and $1.3 \%(n=4)$ of drownings respectively. The cumulative proportion of drownings caused by activities or circumstances not classified by the WHO as drowning deaths was 63.0\% $(n=201)$.

Conclusion \& Learning Outcomes The WHO underestimates the drowning burden in Nigeria by at least 50\%. All-cause drowning codes must be included in mortality statistics to state the true extent of the global drowning crisis. Watercraft accidents are a major cause of drowning in Nigeria; immediate targeted prevention strategies are needed

\section{B.004 FATAL DROWNING IN NIGERIA: A RETROSPECTIVE SURVEY OF NATIONAL NEWSPAPER REPORTS}

\footnotetext{
1,2Anthony Mckeever*, 3,4Shafkat Hossain. 'Liverpool School of Tropical Medicine, Liverpool, UK; ${ }^{2}$ University of Liverpool Medical School, Liverpool, UK; ${ }^{3}$ International Drowning Research Centre, Bangladesh, Mohakhali, Bangladesh; ${ }^{4}$ Centre for Injury Prevention and Research, Bangladesh, Mohakhali, Bangladesh
}

\subsection{6/injuryprev-2021-safety. 155}

Background In 2016, 16,200 people drowned in Nigeria; 22\% of the continental death toll. Yet there are no state or NGO sponsored drowning prevention programmes in Nigeria targeting this unrecognised drowning crisis.

Aim To identify the risks and hazards associated with fatal drowning in Nigeria.

Methods Vanguard news online database was searched for articles reporting fatal drowning incidents between 20092019. Key variables including age, gender and water body were extracted from each article to create a sample of drowning victims. This dataset was then quantitatively analysed to identify hazards and risks associated with drowning.
Results 346 drowning victims were sampled from 98 articles. The male to female drowning ratio was $2.4: 1$. The highest number of drownings were amongst 10 to 19 year-olds; over $50 \%$ of victims were under 20-years-old. Watercraft accidents $(54.9 \%[n=175])$, followed by intentional entry into water bodies $(19.1 \%[n=61])$ were the commonest causes of drowning. Rivers, streams and creeks $(60.6 \%[n=160])$ were the commonest water bodies in which victims drowned. Wells, ponds and water containers collectively accounted for $12.9 \%$ $(n=34)$ of drownings. The ratio of deaths occurring in the wet season compared to the dry season was 3.5:1. 13.0\% $(n=45)$ of drownings were related to flooding or heavy rainfall.

Conclusion \& Learning Outcomes Children and males are particularly vulnerable. In-land water bodies pose a significant threat, with numerous deaths caused by watercraft accidents. Preliminary drowning prevention programmes targeting these vulnerable populations must be established.

\section{C - Strategy, March 25, 2021}

\section{C.001 SUCCESS FACTORS FOR ACHIEVING LONG TERM IMPACT OF A RESEARCH-POLICY PARTNERSHIP}

${ }^{1}$ Samantha Barker*, ${ }^{2}$ Andrea de Silva. 'Institute For Safety, Compensation and Recovery Research (ISCRR), Melbourne, Australia; ${ }^{2}$ WorkSafe Victoria, Geelong, Australia

\subsection{6/injuryprev-2021-safety. 156}

The Institute for Safety, Compensation and Recovery Research (ISCRR) is a unique research-policy partnership between WorkSafe Victoria and Monash University. Having recently celebrated ten years of operation, ISCRR remains focused on delivering high impact research and insights that help to prevent workplace injuries and illness, supporting injured workers to return to health and return to work.

Through a unique approach ISCRR works in partnership with WorkSafe and its stakeholders to co-design and deliver an action research program, with a focus on integrating evidence into decision-making processes across the breadth of WorkSafe activities. This is achieved by remaining flexible and adaptable to changing context and priorities, while also operating within a continuous improvement framework.

This project identifies and shares the learnings over the course of ISCRR's operation. These include key success factors working in partnership to create knowledge and influencing decision-making. Success has included wholesale changes to the research model itself, moving from a mostly research broker function to develop as implementation specialists, and integrating research evidence into the development of new WorkSafe processes, policy and programs, developing new methodologies and research translation techniques. New approaches were developed for synthesising research and practice evidence into useful insights to build a shared understanding of current evidence, and collaboratively interpreting findings and communicating learnings, identifying implications for WorkSafe and the broader sector.

ISCRR's partnership model demonstrates what is required to successfully implement a genuine research collaboration that delivers impacts for the community with applicability to various disciplines and both local and international contexts. 